\title{
Atenção primária à saúde em tempos de pandemia
}

\author{
Primary health care in times of pandemic
}

Atención primaria de salud en tiempos de pandemia

\author{
Cíntia Siqueira Araújo SOARES $S^{(1)}$ \\ Cristina Luiza Ramos da FONSECA ${ }^{(2)}$
}

Recebido: 17 maio 2020

Revisado: 03 jun 2020

Aceito: 01 jul 2020

Autor de correspondência: Cíntia Siqueira Araújo Soares cintia.soares@ifmg.edu.br

Conflito de interesses:

Os autores declaram não haver nenhum interesse profissional ou pessoal que possa gerar conflito de interesses em relação a este manuscrito

\section{Resumo}

O mundo vivencia algo totalmente novo e incerto: a pandemia do Coronavírus (COVID-19). Essa pandemia vem trazendo impactos para a vida da sociedade e, em muitos casos, acarretando às pessoas mudança de hábitos e rotinas, obrigando-as a conviver com incertezas. Em face a isso, surgem questões no campo da gestão da saúde de como não só atuar com cuidados curativos imprescindíveis neste momento, como também atuar em prevenção e educação da população frente à doença. Nesse sentido, este documento busca discutir como a Atenção Primária à Saúde - APS, por meio da Estratégia da Saúde da Família - ESF, pode atuar na luta contra o COVID-19. A partir das reflexões apresentadas neste documento, pode-se concluir que a APS possui um papel fundamental na rede de atenção à saúde e não pode ficar à parte durante a pandemia. Considera-se, entretanto, que regras de prevenção e provimento de equipamentos de proteção individual para todos os colaboradores, na APS, devem ser observados; tal situação para alguns autores, tende a ser exceção, principalmente em locais mais pobres, com baixa qualidade de atenção à saúde. Ressaltase que, caso não se observem as condutas necessárias para se mitigar o risco de infecção para os componentes da equipe do ESF, o funcionamento da Unidade Básica de Saúde UBS pode apresentar mais risco do que auxílio nesta pandemia. É necessário, também, repensar o papel da ESF cuja atuação na saúde é complexa e exige que os profissionais da equipe, como os médicos, enfermeiros, técnicos de enfermagem e agentes comunitários de saúde, sejam capacitados para tanto. Além de treinamentos específicos em relação à atuação nas pandemias, é preciso promover a especialização em saúde da família para todos os componentes da equipe de nível superior.

Descritores: Atenção Primária à Saúde; Estratégia Saúde da Família; Infecções por Coronavírus. 


\begin{abstract}
The world is experiencing something totally new and uncertain: the Coronavirus pandemic (COVID 19). This pandemic directly impacts the life of the society and, in many cases, has caused people to change habits and routines, forcing them to live with uncertainties. Thinking about it, questions arise in the field of health management of how to not only act with curative care essential at this time, but also act in the prevention and education of the population in the face of the disease. Thus, this manuscript seeks to discuss how Primary Health Care - PHC, through the Family Health Strategy ESF, can act in the fight against COVID-19. From the reflections presented in this manuscript, it can be concluded that PHC has a fundamental role in the health care network and cannot be apart during the pandemic. However, the rules of prevention and the provision of personal protective equipment for all employees must be observed, a situation that, for some authors, tends to be an exception in poorer places with low quality of health care. If the conduct necessary to mitigate the risk of infection to the members of the ESF team is not observed, the functioning of the Basic Health Unit - UBS may present more risk than assistance. It is also necessary to rethink the role of the FHS whose performance in health is complex and demands trained professionals. In addition to specific training in relation to acting in pandemics, it is necessary to promote specialization in family health for all members of the higher education team.
\end{abstract}

Keywords: Primary Health Care; Family Health Strategy; Coronavirus Infections.

\title{
Resumen
}

El mundo está experimentando algo totalmente nuevo e incierto: la pandemia de coronavirus (COVID-19). Esta pandemia ha tenido un impacto en la vida de la sociedad y, en muchos casos, ha causado que las personas cambien sus hábitos y rutinas, obligándoles a vivir con incertidumbres. Con esto en mente, surgen preguntas en el campo de la gestión de la salud sobre cómo actuar no solo con una atención curativa esencial en este momento, sino también actuar en la prevención y educación de la población frente a la enfermedad. Así, este manuscrito busca discutir cómo la Atención Primaria de Salud - APS), a través de la Estrategia de Salud Familiar - ESF, puede actuar en la lucha contra COVID-19. A partir de las reflexiones presentadas en este manuscrito, se puede concluir que la APS tiene un papel fundamental en la red de atención médica y no se puede dejar de lado durante la pandemia. Sin embargo, deben observarse las reglas de prevención y la provisión de equipos de protección personal para todos los empleados, una situación que, para algunos autores, tiende a ser una excepción en los lugares más pobres con atención médica de baja calidad. Si no se observa la conducta necesaria para mitigar el riesgo de infección para los miembros del equipo de FHS, el funcionamiento de la Unidad Básica de Salud - UBS puede presentar más riesgo que asistencia. También es necesario repensar el papel del FHS, cuyo desempeño en salud es complejo y requiere que los profesionales del equipo, como médicos, enfermeras, técnicos de enfermería y agentes de salud comunitarios, estén capacitados para hacerlo. Además de la capacitación específica en relación con la actuación en pandemias, es necesario promover la especialización en salud familiar para todos los miembros del equipo de educación superior.

Palabras-claves: Atención Primaria de Salud; Estrategia de Salud Familiar; Infecciones por Coronavirus.

\section{Introdução}

O mundo vivencia algo totalmente novo e incerto: a pandemia do Coronavírus (COVID-19). Essa pandemia vem trazendo impactos para a vida da sociedade e, em muitos casos, acarretando às pessoas mudança de hábitos e rotinas, obrigando-as a conviver com incertezas. Em face a isso, surgem questões no campo da gestão da saúde, como a de não só atuar com cuidados curativos, imprescindíveis neste momento, como também atuar na prevenção e na educação da população frente à doença. Nesse sentido, há vários aspectos 
que precisam ser focalizados e, então, neste documento, se busca discutir como a Atenção Primária à Saúde - APS, por meio da Estratégia da Saúde da Família - ESF, pode agir na luta contra o COVID-19.

Para iniciar o debate, é preciso retomar a mudança do foco assistencial de curativo para preventivo, proposta pelo Sistema Único de Saúde - SUS, ${ }^{1}$ tornando a atenção primária à saúde central na organização da rede de atenção à saúde. ${ }^{2}$ Assim, seu papel primordial na reorganização da assistência ${ }^{3}$ é o de buscar aumento de resolutividade com impacto na saúde pública, aprimorando-se a relação custo-efetividade. ${ }^{4}$

\section{Desenvolvimento}

A APS tem como missão realizar ações preventivas e curativas de forma regionalizada, contínua e sistematizada; trabalhando junto com as comunidades. ${ }^{5}$ No Brasil, a APS foi implementada por meio do Programa Saúde da Família, atualmente, Estratégia da Saúde da Família. A ESF estabeleceu a adstrição de usuários, fixando o território de abrangência e focando a família. ${ }^{6}$ O programa determinou cobertura universal de saúde, melhorando acesso e satisfação da população e qualidade da atenção à saúde.7

Por conseguinte, a equipe da ESF possui diversas atribuições, dentre elas: atuar no processo de territorialização da saúde por meio de busca ativa e notificação de doenças, prezando pela qualidade dos dados. Ademais, deve identificar situações de vulnerabilidade e risco, acompanhando famílias e indivíduos de modo a manter a fidedignidade do diagnóstico da situação de saúde da comunidade por meio da avaliação combinada da equipe multidisciplinar existente na ESF. ${ }^{8}$

É importante salientar que, na ESF, esta equipe multiprofissional é composta por, no mínimo, um médico generalista ou especialista em saúde da família ou em família e comunidade, enfermeiro generalista ou especialista em saúde da família, auxiliar ou técnico de enfermagem e agentes comunitários de saúde (ACS). ${ }^{9}$ Em algumas equipes, podem também ser adicionados profissionais de Saúde Bucal, tais como cirurgião-dentista generalista ou especialista em saúde da família, auxiliar e/ou técnico em Saúde Bucal. ${ }^{10}$ Além disto, em algumas Unidade Básica de Saúde, UBSs, há o Núcleo de Apoio à Saúde da família, composto por profissionais que complementam o atendimento da comunidade como, por exemplo, psicólogos, fisioterapeutas, médicos veterinários, farmacêutico, psiquiatra, dentre outros.

Toda essa equipe deve seguir os preceitos do SUS, citados anteriormente, e agir para a efetivação das tarefas da ESF. Nesse sentido, o vínculo com os usuários e a comunidade é um ponto relevante. Ele deve ser construído por meio da escuta qualificada, resolubilidade 
dos encaminhamentos e atendimentos, sejam eles na UBS ou em domicílio, buscando estimular a autonomia das pessoas quanto aos cuidados de saúde. Dessa forma, a ESF deve atuar também em ações educativas, que promovam a saúde, e a participação da comunidade na gestão do serviço. ${ }^{8}$ Focando na atenção integral, equânime e contínua, a ESF deve, ainda, estabelecer ações de prevenção e promoção da saúde. ${ }^{11}$

\section{Discussão}

Considerando as atribuições citadas anteriormente, ${ }^{12}$ é possível afirmar que a ESF possui uma ampla atuação que envolve ações de:

a) atenção à saúde, continuada, integral, avaliando os sintomas do indivíduo, o histórico familiar e a situação de saúde da comunidade;

b) prevenção e promoção à saúde, por meio de ações educativas e estímulo a hábitos saudáveis, como alimentação adequada e exercícios físicos; e

c) vigilância epidemiológica, por meio da identificação e notificação de doenças.

Desse modo, a ESF possui um papel preponderante para a manutenção da saúde da população. Por isso, é necessário pensar no seu papel em situações de emergência de saúde; afinal é onde a atenção primária tem uma atuação mais presente e há ganhos significativos na condição de saúde da população, ${ }^{13,14}$ especialmente em epidemias. ${ }^{15,16}$

Diante disto, as atividades rotineiras da ESF, como vacinação, consultas agendadas, acompanhamento dos grupos de riscos e de doenças crônicas, devem ser mantidas com as adequações necessárias. Essas adequações envolvem, por exemplo, garantir Equipamentos de Segurança Individual - EPI a todos os profissionais da saúde, atendimento em locais diferenciados para cada atividade, ${ }^{17}$ higienização constante dos locais, trabalho com agendamentos, quando possível, para evitar aglomerações, utilização do atendimento por telefone ${ }^{18}$ garantindo, assim, a segurança da população quanto à possibilidade de contágio por vírus na UBS.

Assim, é necessário repensar os processos de trabalhos e de atuação de cada profissional que compõe a equipe de modo a evitar que sejam veículos de transmissão do vírus. Para tanto, deve-se avaliar estratégias como os atendimentos a distância, com a utilização da tecnologia.

Consultas e acompanhamento dos grupos de riscos e de doenças crônicas podem ser realizados com o apoio da tecnologia, por meio da internet e, especialmente, por ligações telefônicas, considerando a realidade de infraestrutura de cada UBS. A carência de internet de banda larga e de câmeras filmadoras pode dificultar a realização de tele consultas, limitando a atuação na avaliação a distância do paciente. ${ }^{15}$ Nesse processo, o ACS pode se 
tornar uma peça chave, como responsável pelas ligações telefônicas constantes para acompanhamento da condição de saúde da comunidade, posto que, já possui, como principais atividades, a identificação de situações de risco, a orientação de famílias e comunidade e encaminhamento de casos e situações de risco identificados aos outros membros das equipes de saúde. ${ }^{19}$

Esse papel do ACS, como citado no parágrafo anterior, será fundamental nessa pandemia tanto para controle dos grupos de risco e teleatendimento das consultas para casos diversos, inclusive de sintomáticos respiratórios, como também para a conscientização das medidas a serem tomadas para diminuição da disseminação da COVID-19, fazendo com que a população se sinta assistida e apoiada frente à situação, ${ }^{20}$ visto que fortalecer os cuidados domiciliares se torna essencial para a organização das ações de ACS e agentes de endemias. $^{21}$

Ademais, a ESF, como dito anteriormente, possui a função de garantir o acesso da população a uma saúde integral, coordenada e longitudinal ${ }_{i}^{15}$ portanto, é preciso a manutenção das ações próprias da atenção primária, ${ }^{21}$ em especial o monitoramento dos casos crônicos, afinal as pessoas irão continuar tendo pressão alta, diabetes, tuberculose, dentre outros. Desse modo, não é possível negar o atendimento a essas pessoas, pois, além de serem de um grupo de risco, é possível que a mortalidade e a morbidade desse grupo aumentem em função da diminuição das ações preventivas e curativas no período de pandemia.

Nesse contexto, é importante ainda debater as ações preventivas que podem ser realizadas pelas equipes da ESF em tempos de pandemia. Deve-se enfatizar que um dos pilares dessa estratégia é o envolvimento com a família e a comunidade por meio do qual se busca identificar as especificidades, nuances e complexidades relativas à saúde da população adstrita. Conhecendo esta população, é possível atuar de forma ativa e adequada em relação a cada realidade.

Dentre as ações preventivas, os profissionais das equipes básicas devem buscar estratégias de redução de aglomerações nas unidades básicas para evitar a transmissão do Coronavírus, nessa pandemia, além de construir táticas para facilitar a implantação de medidas de higiene e proteção social e familiar, reforçando o isolamento e a quarentena, quando aplicáveis.

Para tanto, é possível que a gestão da atenção primária em saúde do município busque construir parcerias para realização de pequenas gravações de áudio e vídeo que possam ser divulgadas por aplicativos de mensagens, como por exemplo o WhatsApp $\AA$, de modo a que toda a população tenha acesso a informações importantes; ou, ainda, divulgar 
vídeos já existentes e disponíveis na web cujo conteúdo tenha sido validado por algum profissional de saúde. Essas gravações podem tratar dos seguintes temas:

1. Máscaras: ensinar como fazê-las; como utilizá-las; a importância do seu uso.

2. Isolamento social: o que é; sua influência na pandemia; alternativas para superar a solidão; criação de rede de apoio na própria comunidade por telefone, aproximando as pessoas, sem necessidade de contato físico.

3. Contágio: como se dá o contágio; como funciona a curva de contágio, esclarecendo que as ações de hoje apenas terão efeito após duas semanas ou mais; ações de prevenção do contágio; ações de higiene pessoal que minimizam o risco de contágio, como ensinar a lavar as mãos, procedimentos de desinfecção ao chegar em casa e dos produtos adquiridos; etiqueta da tosse e outros pertinentes.

4. Atividade física: envio de vídeos ou links de vídeos com atividades físicas que sejam compatíveis como cada grupo de pessoas (separando a população por faixa etária e condição de saúde).

Finalmente, quanto a procedimentos de vigilância em saúde e epidemiológica, conforme atribuição da equipe ${ }^{12}$ o papel da ESF na identificação de agravos ainda permanece importante, especialmente durante uma pandemia, em que dados precisos são imprescindíveis. ${ }^{22}$ Estes dados vão auxiliar as autoridades em saúde, como gestores em níveis federal, estadual e municipal, a tomarem as decisões pertinentes, considerando a realidade epidemiológica de cada localidade. A partir dessa avaliação, é possível definir políticas públicas, tais como medidas de lockdown ou flexibilização do isolamento, com funcionamento de atividades econômicas.

A ESF pode também se responsabilizar por auxiliar na coleta do material para testagem em massa ou realizar testes rápidos confiáveis, como foi feito, por exemplo, nos municípios de Canaã dos Carajás ${ }^{18}$ e Florianópolis $^{23}$ desde que observado o cuidado com a proteção da equipe e da população, utilizando-se EPIs específicos, treinando-se efetivamente equipes e definindo a atuação de cada cargo.

A ESF pode ainda assumir papel resolutivo frente aos casos leves e de identificação precoce, orientando isolamento e monitorando a saúde do paciente, remotamente, como foi feito no município de Nova Lima, adotando-se estratégias como o monitoramento por telefone (Alô Saúde). ${ }^{24}$ Quanto aos casos graves, a equipe pode encaminhá-los corretamente para o restante da rede ou, ainda, enviá-los para o monitoramento de um profissional médico ou estudante de medicina, como aconteceu no município de Rio Branco. ${ }^{25}$

Ressalta-se, entretanto, que, se for preciso atendimento presencial, a UBS deve utilizar medidas que minimizem a possibilidade de contaminação dos profissionais, tais como uso de $\mathrm{EPI}^{26}$ ou deve ser capaz de se adaptar para receber o caso identificado sem pôr em risco 
o restante da comunidade. Essas adaptações podem ser operacionalizadas por meio da reorganização da rede, designando uma UBS para receber todos os $\operatorname{casos}^{18}$, ou construindo tendas externas para atendimento de sintomáticos ${ }^{27}$, ou definindo outra porta de entrada para os possíveis casos de COVID-19. ${ }^{26}$

\section{Conclusão}

A partir dessas reflexões, pode-se concluir que a APS possui um papel fundamental na rede de atenção à saúde e não pode ficar à parte durante a pandemia, mas é preciso observarem-se as regras de prevenção e prover EPI para todos os colaboradores, realidade que tende a ser exceção em locais mais pobres com baixa qualidade de atenção à saúde. ${ }^{28}$ Caso não se observem as condutas necessárias para se mitigar o risco de infecção aos componentes da equipe do ESF, o funcionamento da UBS pode apresentar mais risco do que auxílio em pandemia. ${ }^{27}$

É necessário, ainda, repensar o papel da ESF cuja atuação na saúde não é simples e exige que profissionais sejam capacitados para nela trabalhar. Além de treinamentos específicos da ESF em relação à atuação nas pandemias, é preciso promover a especialização em saúde da família para todos os seus componentes de nível superior.

Finalmente, destaca-se que, ao final de uma pandemia, o funcionamento da ESF não pode o mesmo de antes. É necessário condensar todas as lições e elaborar um plano de enfrentamento para futuras pandemias, fortalecendo a atenção primária e o vínculo com a comunidade, de forma que a Unidade funcione como uma barreira para evitar o aumento da propagação da doença. ${ }^{29}$ Não se deve esquecer que o plano deve considerar peculiaridades de cada região e comunidade, sendo, preferencialmente, construído com a comunidade, em conformidade com a diretriz de promover a autonomia da população. Alguns pontos, entretanto, são consenso em relação às lições aprendidas sobre o enfrentamento de uma pandemia. O modelo hospitalocêntrico não contribui para o combate de uma pandemia e que é necessária uma real aproximação com a comunidade. ${ }^{30}$ Nesse sentido, é preciso valorizar a APS, em especial seus profissionais, e estimular o engajamento da população adstrita, inclusive por meio dos Conselhos de Saúde. ${ }^{24}$

\section{Referências}

1. Vasconcelos RNC, Ruiz EM. Formação de médicos para o SUS: a integração ensino e saúde da família - revisão integrativa. Rev Bras Educ Med. 2015;39(4):630-8. https://doi.org/10.1590/1981-52712015v39n4e02772014. 
2. Mendes EV. Desafios do SUS. Brasília, DF: CONASS; 2019 [citado em 3 maio 2020]. Disponível em: http://www.conass.org.br/biblioteca/desafios-do-sus/.

3. Turci MA, organizador. Avanços e desafios na organização da Atenção de saúde em Belo Horizonte. Belo Horizonte: Secretaria Municipal de Saúde; 2008.

4. Ministério da Saúde (BR). Ministério da Saúde anuncia avanços na Política Nacional de Atenção Básica, 2017 [citado em 22 mar. 2020]. Disponível em: https://www.youtube.com/watch?v=--4ik-LkF98\&feature=youtu.be.

5. Pereira IB, Lima JCF, organizadores. Dicionário da educação profissional em saúde. 2a ed. Rio de Janeiro: EPSJV; 2008 [citado em 5 maio 2020]. Disponível em: https://www.arca.fiocruz.br/bitstream/icict/25955/2/Livro\%20EPSJV\%20008871.pdf.

6. Ministério da Saúde (BR). Portaria n. 2.488, de 21 de outubro de 2011. Aprova a Política Nacional de Atenção Básica, estabelecendo a revisão de diretrizes e normas para a organização da Atenção Básica, para a Estratégia Saúde da Família (ESF) e o Programa de Agentes Comunitários de Saúde (PACS). Brasília, DF: Diário Oficial da União; 2011 [citado em 1 maio 2020]. Disponível em: http://bvsms.saude.gov.br/bvs/saudelegis/gm/2011/prt2488_21_10_2011.html.

7. Barbosa LG, Damasceno RF, Silveira DMMLD, Costa SDM, Leite MTDS. Recursos Humanos e Estratégia Saúde da Família no norte de Minas Gerais: avanços e desafios. Cad Saude Colet. 2019;27(3);287-94. http://dx.doi.org/10.1590/1414-462x201900030084.

8. Figueiredo EN. A estratégia saúde da família na atenção básica do SUS. 2015 [citado em 5 maio 2020]. Disponível em:

https://www.unasus.unifesp.br/biblioteca_virtual/esf/2/unidades_conteudos/unidade05/un idade05.pdf.

9. Ministério da Saúde (BR). Saúde da família. Brasília, DF: MS; 2017 [citado em 23 jun. 2020]. Disponível em: https://www.saude.gov.br/artigos/772-acoes-e-programas/saude-dafamilia/41285-saude-dafamilia\#: :text=A\%20Estrat\%C3\%A9gia\%20Sa\%C3\%BAde\%20da\%20Fam\%C3\%ADlia\%20(E SF)\%20\%C3\%A9\%20composta\%20por\%20equipe,agentes\%20comunit\%C3\%A1rios\%20de \%20sa\%C3\%BAde\%20(ACS).

10. Ministério da Saúde (BR). Estratégia da Saúde da Família (ESF). Brasília, DF: MS; 2020 [citado em 5 maio 2020]. Disponível em: https://www.saude.gov.br/acoes-eprogramas/saude-da-familia. 
11. Ministério da Saúde (BR). Portaria n. 2.436, de 21 de setembro de 2017. Aprova a Política Nacional de Atenção Básica, estabelecendo a revisão de diretrizes para a organização da Atenção Básica, no âmbito do Sistema Único de Saúde (SUS). Brasília, DF: 2017 [citado em 5 maio 2020]. Disponível em:

https://bvsms.saude.gov.br/bvs/saudelegis/gm/2017/prt2436_22_09_2017.html.

12. Ministério da Saúde (BR). Estratégia da Saúde da Família (ESF). Brasília, DF: MS; 2020 [citado em 5 maio 2020]. Disponível em: https://www.saude.gov.br/acoes-eprogramas/saude-da-familia/sobre-o-programa.

13. Macinko J, Starfield B, SHI L. The contribution of primary care systems to health outcomes within Organization for Economic Cooperation and Development (OECD) countries, 1970 1998. Health Serv Res. 2003 Jun;38(3):831-65, 2003. https://doi.org/10.1111/14756773.00149.

14. Basu S, Berkowitz SA, Phillips RL, Bitton A, Landon BE, Phillips RS. Association of primary care physician supply with population mortality in the United States, 2005-2015. JAMA Intern Med. 2019;179(4):506-14. https://doi.org/10.1001/jamainternmed.2018.7624

15. Minué S. Contra el coronavirus, más Atención Primaria que nunca. Actualización en Medicina de Familia. c2020 [citado em 10 abr. 2020]. Disponível em: https://amfsemfyc.com/web/article_ver.php?id=2626.

16. Minué S. La pandemia COVID-19: lo que hemos aprendido hasta ahora desde España. APS Rev. 2020 abr. 15;2(1):28-32. https://doi.org/10.14295/aps.v2i1.66.

17. Guimarães FG, Carvalho TML, Bernardes RM, Pinto JM. A organização da atenção Primária à Saúde de Belo Horizonte no enfrentamento da pandemia Covid 19: relato de experiência. APS Rev. 2020 jun. 9;2(2):74-82. https://doi.org/10.14295/aps.v2i2.128.

18. Vale EP, Rodrigues GM, Costa DP, Queiroz JM, Lima DG, Mereiros LPF, et al. Reorganização da Rede de Atenção à Saúde para o enfrentamento da COVID-19 no município de Canaã dos Carajás, Pará. APS Rev. 2020 jun. 9;2(2):83-90. https://doi.org/10.14295/aps.v2i2.101.

19. Tomaz JBC. O agente comunitário de saúde não deve ser um "super-herói". Interface (Botucatu). 2002;6(10):84-7. https://doi.org/10.1590/S1414-32832002000100008.

20. Nedel FB. Enfrentando a COVID-19: APS forte agora mais que nunca! APS Rev. 2020 abr. 15;2(1):11-16. https://doi.org/10.14295/aps.v2i1.68.

21. Medina MG. Desafios da APS no SUS no enfrentamento da Covid-19. Seminário Virtual Rede APS Abrasco, 2020 [citado em 5 maio 2020]. Disponível em: 
https://redeaps.org.br/wp-content/uploads/2020/04/Relatorio-Rede-APS-

ISSN 2179-6750

_Semina\%CC\%81rio-APS-no-SUS-e-Covid-16-Abril-2020-final.pdf.

22. Leon DA, Shkolnikov VM, Smeeth L, Magnus P, Pechholdová M, Jarvis CI. COVID-19: a need for real-time monitoring of weekly excess deaths. Lancet. 2020 May;395(10234):e81. https://doi.org/10.1016/S0140-6736(20)30933-8.

23. Silveira JPM, Zonta R. Experiência de reorganização da APS para o enfrentamento da COVID-19 em Florianópolis. APS Rev. 2020 jun. 9;2(2):91-6.

https://doi.org/10.14295/aps.v2i2.122.

24. Fernandez MV, Castro DM, Fernandes LMM, Alves IC. Reorganizar para avançar: a experiência da Atenção Primária à Saúde de Nova Lima/MG no enfrentamento da pandemia da Covid-19. APS Rev. 2020 jun. 9;2(2):114-21. https://doi.org/10.14295/aps.v2i2.84.

25. Silveira RP, Leal O, Soares PLS, Cruz LF, Modesto IDM, Batista LN, et al. Telemonitoramento da COVID-19 com participação de estudantes de medicina: experiência na coordenação do cuidado em Rio Branco, Acre. APS Rev. 2020 jun. 9;2(2):151-61. https://doi.org/10.14295/aps.v2i2.121.

26. Mendonça CS, Rosset I, Gonçalves MR, Bastos CGM, Medeiros AF, Dias AV, et al. Resposta assistencial de um serviço docente assistencial de APS à pandemia da COVID-19. APS Rev. 2020 abr. 15;2(1):33-7. https://doi.org/10.14295/aps.v2i1.63.

27. Tasca R, Massuda A. Estratégias para reorganização da Rede de Atenção à Saúde em resposta à Pandemia COVID-19: a experiência do Sistema de Saúde Italiano na região de Lazio. APS Rev. 2020 abr. 15;2(1):20-7. https://doi.org/10.14295/aps.v2i1.65.

28. Roder-De Wan S. Health system quality in the time of COVID-19. Lancet Glob Health. 2020 jun.;8(6):e738-9. https://doi.org/10.1016/S2214-109X(20)30223-0.

29. Tasca, R. Desafios da APS no SUS no enfrentamento da Covid-19. Seminário Virtual Rede APS Abrasco, 2020 [citado em 5 maio 2020]. Disponível em: https://redeaps.org.br/wpcontent/uploads/2020/04/Relatorio-Rede-APS-_Semina\%CC\%81rio-APS-no-SUS-e-Covid16-Abril-2020-final.pdf.

30. Nacoti M, Ciocca A, Giupponi A, Brambillasca P, Lussana F. Pisano $M$, et al. At the epicenter of the Covid-19 pandemic and humanitarian crises in Italy: changing perspectives on preparation and mitigation. NEJM Catalyst. 2020 Mar 21 [citado em 24 jun. 2020];1(2). Disponível em: https://catalyst.nejm.org/doi/full/10.1056/CAT.20.0080. 
Cíntia Siqueira Araújo Soares | ORCiD: 0000-0002-8457-4997

Mestre em Administração pela Universidade Federal de Minas Gerias - UFMG, Especialista em Docência no Ensino Superior e Gestão de Recursos Humanos, Graduada em Administração pela Faculdade de Ciências Econômicas e Administrativas de Divinópolis -FACED. Professora Substituta do Instituto Federal de Minas Gerais - Campus Bambuí.

Cristina Luiza Ramos da Fonseca | ORCiD: 0000-0002-5246-6609

Doutoranda em Administração pela Universidade Federal de Minas Gerais, Mestre em Gestão da Saúde pela Escola Nacional de Saúde Pública - ENSP/Fiocruz, RJ. Graduada em Relações Internacionais pela Pontifícia Universidade Católica - PUC/MG. Analista de Gestão em Saúde do Instituto René Rachou - IRR/Fiocruz Minas. Belo Horizonte, MG, Brasil 